\title{
La sensibilità del consumatore a comportamenti Recered aziendali eticamente scorretti
}

Giuseppe Bertoli - Bruno Busacca - Alessandro Calzetti

\begin{abstract}
Obiettivi del paper: Il paper analizza l'effetto dellalterazione dei dati inerenti al prodotto e alla responsabilità sociale dell'impresa sull'intenzione d'acquisto del consumatore e come tali tipologie di alterazione interagiscono con la brand image e l'autostima individuale.

Metodologia: La ricerca si fonda su un esperimento between subjects condotto su un campione di 200 consumatori. Il disegno fattoriale prevede tre variabili indipendenti: lalterazione dei dati di prodotto (product sophistication vs product social responsibility); la brand image (forte vs debole); e l'autostima dell'individuo (alta vs bassa). La variabile dipendente è costituita dall'intenzione d'acquisto del consumatore.

Risultati: I dati ottenuti dimostrano che i consumatori sono influenzati in misura maggiormente negativa dall'alterazione dei dati di product sophistication che non da quella concernente la product social responsibility. In secondo luogo, l'intenzione d'acquisto del consumatore in presenza di quest'ultimo tipo di alterazione risulta più elevata quando l'impresa ha saputo sviluppare associazioni forti, favorevoli e uniche alla propria marca. In terzo luogo, $i$ dati dimostrano che tale intenzione si accresce ulteriormente se, in presenza del medesimo tipo di alterazione dei dati, a una forte immagine di marca si accompagna unialta autostima individuale.

Limiti della ricerca: Nell'indagine non è stato considerato il livello di coinvolgimento psicologico del consumatore nei confronti delle tematiche etiche in generale e di alcune di esse in particolare. Inoltre, non è stato valutato l'impatto della fedeltà alla marca e della percezione della sua unicità. In terzo luogo, si è esaminata un'unica variabile psicografica.

Implicazioni pratiche: I risultati ottenuti confermano la centralità delle informazioni sul prodotto, l'importanza dell'immagine di marca e l'opportunità di segmentare $i$ consumatori sulla base del grado di autostima. Emergono inoltre significative implicazioni per quanto concerne la tutela dell'interesse collettivo.

Originalità del paper: Il tema dell'impatto dellalterazione delle due tipologie di informazioni concernenti il prodotto aziendale e della loro interazione con la brand image e lautostima non ha sin qui ricevuto attenzione.
\end{abstract}

Parole chiave: alterazione dei dati; corporate social responsibility; immagine di marca; autostima 


\section{sinergie Consumer sensitivity to unethical corporate behavior}

Purpose of the paper: The paper analyzes: a) the effect of data alteration related to product sophistication and to product social responsibility on the consumer purchase intention; $b$ ) how these types of alteration interact with the brand image and individual self-esteem.

Methodology: The research is based on a between subjects experiment conducted on a sample of 200 consumers. The factorial design includes three independent variables: falsification of product data (product sophistication vs. product social responsibility); the brand image (strong vs. weak); and the individual self-esteem (high vs. low). The dependent variable is the consumer purchase intention.

Findings: The data obtained show that consumers are more negatively influenced by the alteration of data concerning product sophistication than product social responsibility. Secondly, the consumer purchase intention in the presence of the latter type of alteration is higher when the company has been able to develop a strong brand image. Thirdly, purchase intention is further enhanced if, in the presence of the same type of falsification of data, a strong brand image is associated to a high individual self-esteem.

Research limits: The survey did not consider the level of psychological involvement of the consumer in relation to ethical issues or the product category. Furthermore, the impact of brand loyalty and the perception of its uniqueness was not assessed. Thirdly, a single psychographic variable was examined.

Practical implications: The results obtained confirm the centrality of the information on the product, the importance of the brand image and the opportunity to segment consumers based on the degree of self-esteem. There are also significant implications for the protection of collective interests.

Originality of the paper: So far, the impact of different types of product data falsification on consumer purchase intention and their interaction with the brand image and the individual self-esteem has not received adequate attention.

Key words: alteration of product data; corporate social responsibility; brand image; self-esteem

\section{Introduzione}

Lattenzione alla responsabilità sociale è sempre più al centro delle strategie aziendali (ad es. Porter e Kramer, 2011; Perrini et al., 2011; Tencati e Pogutz, 2015; Rasche et al., 2017) e numerosi studi evidenziano che, a parità di altre condizioni, i consumatori esprimono una valutazione peggiore nei confronti delle imprese che pongono in atto comportamenti eticamente scorretti (Carrigan e Attalla, 2001; Sen e Bhattacharya, 2001; De Pelsmacker et al., 2005; Mohr e Webb, 2005; Newholm e Shaw, 2007).

Fra i comportamenti riprovevoli che hanno ottenuto ampio risalto dalla cronaca in tempi relativamente recenti, è possibile ricordare il caso di una nota compagnia accusata per alcuni dispositivi telefonici che non rispettavano la durata della batteria o i gigabyte dichiarati, o quello di un'importante casa automobilistica che ha comunicato dati sulle emissioni inquinanti di alcune sue vetture difformi da quelli reali o, ancora, quello 
di un rinomato produttore di abbigliamento per il quale sono emerse situazioni di maltrattamento degli animali da cui è ottenuta una materia prima rilevante per la sua produzione.

In questi e in altri casi, il comportamento eticamente scorretto, ma non di rado anche illegale, attiene alla cosiddetta alterazione delle informazioni (Hunt et al., 1984; Ferrell e Gresham, 1985; Hunt e Chonko, 1985), la quale si sostanzia nellomissione di informazioni rilevanti o nella diffusione di dati non veritieri. Tale condotta riguarda sovente il prodotto, nel senso che le imprese possono omettere la comunicazione di talune caratteristiche dellofferta o dei sottostanti processi aziendali oppure comunicare caratteristiche superiori o comunque diverse da quelle realmente possedute (Plank e Teichmann, 2018), al fine di superare la concorrenza, incrementare le vendite e migliorare la propria redditività.

Al di là degli orientamenti comportamentali dichiarati, la reale consapevolezza dei consumatori verso le condotte socialmente responsabili delle aziende sembra essere in linea generale non elevata e, anche laddove presente, non pare incidere in misura rilevante sulle effettive decisioni di acquisto. I criteri dichiarati solitamente più importanti risultano infatti: il prezzo, la qualità, la familiarità con la marca e l'immagine di quest'ultima (Carrigan e Attalla, 2001; De Pelsmacker et al., 2005; Mohr e Webb, 2005).

Peraltro, nei casi di consapevolezza del consumatore, la ricerca evidenzia che un comportamento eticamente scorretto esercita un impatto più forte sull'immagine dell'impresa rispetto a uno positivo (Folkes e Kamins, 1999; Sen e Bhattacharya, 2001): è cioè probabile che un consumatore non migliori il giudizio su un'azienda che promuove la responsabilità sociale, ma che, al contrario, osteggi un'impresa che mette in atto comportamenti non etici. Solo i consumatori che ricercano attivamente informazioni sembrano comunque disposti a passare da una marca positivamente valutata a una di minor valore ma che reputano eticamente più attenta (Auger et al., 2003).

Sulla sensibilità del consumatore alle informazioni relative all'eticità dei comportamenti aziendali agiscono numerose variabili, legate sia all'impresa che all'individuo. Con riferimento alle prime, la letteratura ha approfondito in particolare il ruolo della marca, evidenziando come il consumatore: a) valuti ancora più positivamente una marca con la quale si identifica e che pone in atto azioni di CSR; b) sia influenzato meno negativamente da un'informazione concernente un comportamento eticamente scorretto ascrivibile a una marca alla quale è fedele (Sen e Bhattacharya, 2001).

In relazione alle variabili individuali, la letteratura ha invece indagato tanto quelle di natura socio-demografica, evidenziandone il limitato impatto, quanto quelle - più significative - di natura psicografica. Le variabili socio-demografiche non permettono di identificare con precisione un "consumatore etico": studi passati hanno dimostrato che l'età, il sesso o il reddito, ad esempio, non sono discriminanti (Roberts, 1996; Dickson, 2001), mentre altri studi hanno evidenziato risultati non convergenti (Auger et al., 2003; De Pelsmacker et al., 2005). Varie ricerche hanno invece riscontrato la possibilità di individuare "i consumatori etici" e il loro comportamento di acquisto mediante le variabili psicografiche (Anderson e Cunningham, 1972; Cowe e Williams, 2000; Dickson, 2001;
Giuseppe Bertoli

Bruno Busacca

Alessandro Calzetti

La sensibilità del

consumatore a

comportamenti aziendali

eticamente scorretti 
sinergie Vol. 37, Issue 3, 2019

Huttel et al., 2018; Tey et al., 2018). In particolare, i valori - intesi come princìi astratti che riflettono il self-concept di un individuo - orientano le convinzioni in merito al fatto che un determinato comportamento possa essere desiderabile o meno e guidano le decisioni relative alletica (Dickson, 2000).

Il presente lavoro intende contribuire agli studi sulle reazioni dei consumatori ai comportamenti non etici delle aziende introducendo la distinzione tra alterazione dei dati di product sophistication e di product social responsibility, analizzando quale tra esse incida maggiormente sull'intenzione di acquisto del consumatore, per poi indagare l'effetto della loro interazione con l'immagine di marca e con l'autostima dell'individuo, componente fondamentale del self concept. Più precisamente, il contribuito teorico aggiuntivo rispetto alla letteratura sul tema consiste nellesaminare se e come l'immagine di marca possa attutire l'effetto negativo dell'alterazione dei dati, a seconda che questi siano riferiti alla qualità e alla funzionalità del prodotto o alla sua responsabilità sociale. Rispetto a tale interazione, si approfondisce poi l'effetto dell'autostima, che - influenzando direttamente il self concept del consumatore - rappresenta una variabile in grado di incidere anche sulle reazioni a comportamenti non etici.

Il lavoro è così strutturato: il paragrafo 2, alla luce dell'analisi della letteratura, definisce le ipotesi di ricerca che ci si prefigge di verificare; il paragrafo 3 illustra il disegno sperimentale, le scale di misurazione utilizzate e la metodologia d'indagine; il paragrafo 4 sintetizza i risultati ottenuti. Infine, nelle conclusioni vengono discusse le principali implicazioni teoriche e manageriali dell'analisi svolta.

\section{Le ipotesi di ricerca}

I contributi teorici su cui si fondano le ipotesi di ricerca sono riconducibili a tre filoni di indagine, concernenti:

- l'influenza che le associazioni cognitive legate alle abilità dell'impresa e alla sua responsabilità sociale possono esercitare sul consumatore;

- il ruolo dell'immagine di marca nel processo di scelta e i meccanismi alla base dell'inerzia cognitiva da essa generata;

- l'impatto del self concept sul comportamento dell'individuo, con particolare riferimento alle fondamentali dimensioni di tale costrutto.

Con riferimento al primo filone, Brown e Dacin (1997) definiscono le associazioni di corporate ability, come quelle "related to the company's expertise in producing and delivering its outputs", mentre le associazioni di corporate social responsibility, riflettono "the organization's status and activities with respect to its perceived societal obbligations". Gli autori evidenziano come la prima tipologia di associazioni incida in via diretta sulla valutazione degli attributi del prodotto da parte del consumatore, fra i quali la cosiddetta "product sophistication", intesa come la misura in cui il prodotto incorpora i più recenti avanzamenti tecnologici. Le associazioni del secondo tipo, invece, influenzano in via diretta la percezione della marca e/o dell'impresa, rafforzandone l'attrattività e l'affidabilità, ma non direttamente gli attributi del prodotto. Inoltre, il recente studio di Johnson 
et al. (2018) dimostra che le associazioni di corporate social responsibility prevalgono su quelle di corporate ability quando il consumatore valuta l'esperienza di consumo, mentre si verifica il contrario allorché oggetto di considerazione sono gli attributi di prodotto.

Venendo al tema oggetto di indagine nel presente lavoro, è possibile, dunque, distinguere l'alterazione dei dati di product sophistication da quella relativa ai dati sulla product social responsibility. Mentre la prima si verifica quando il prodotto presenta differenze rispetto agli attributi, alla qualità, all'innovazione, alle performance o alle funzionalità comunicate dall'impresa, la seconda ha invece luogo quando il prodotto non è conforme alla responsabilità sociale da essa dichiarata (con riferimento ai diritti umani, alle condizioni di lavoro, al benessere animale, ambientale ecc.).

Benchéanchele associazioni di productsocial responsibility siano ritenute importanti dai consumatori al fine di valutare l'impresa e/o la sua offerta, sono relativamente pochi quelli in grado di ricordare le caratteristiche etiche possedute dai prodotti acquistati o comunque considerati nel processo di scelta. Ben più numerosi sono coloro che rammentano con precisione la marca e le principali caratteristiche tecniche (Auger et al., 2003). Ciò dimostra che, per i consumatori, hanno maggior importanza le associazioni di product sophistication che non quelle di product social responsibility, in particolare al momento dell'acquisto (Berens et al., 2007). Pertanto ci si attende che, nel caso in cui l'alterazione dei dati riguardi il rispetto delle "societal obbligations", gli individui siano meno inclini a penalizzare l'impresa protagonista di tale violazione etica.

Alla luce di quanto osservato, è possibile avanzare la seguente ipotesi di ricerca:

H1) l'intenzione di acquisto del consumatore è più elevata se l'alterazione dei dati riguarda la product social responsibility anziché la product sophistication.

Come evidenziato da numerosi studi nellambito del secondo filone di indagine richiamato in apertura del paragrafo, in molte situazioni l'immagine della marca rappresenta il principale criterio di scelta utilizzato dal consumatore (De Pelsmacker, 2005, p. 375). Essa esercita un impatto particolarmente rilevante sulla percezione di qualità dell’offerta, sul valore e sull'intenzione d'acquisto degli individui (Dodds et al., 1991). L'immagine sintetizza l'insieme delle associazioni nei confronti della marca presenti nella memoria del consumatore (Aaker, 1995). Se tali associazioni sono forti, favorevoli e uniche (Keller, 2012) e se vengono correttamente comunicate, esse aiutano a stabilire un efficace posizionamento del brand e, quindi, a svolgere un ruolo fondamentale nella costruzione del valore della marca nel lungo periodo.

Un brand la cui immagine positiva è ben radicata nella mente del consumatore o nel quale questi si identifica può risultare più resiliente rispetto alla diffusione di notizie negative sullazienda alla quale fa capo (Mohr e Webb, 2005; Oberseder et al., 2011). In questottica, anche di fronte alla conoscenza di comportamenti eticamente scorretti, diversi
Giuseppe Bertoli

Bruno Busacca

Alessandro Calzetti

La sensibilità del

consumatore a

comportamenti aziendali eticamente scorretti 
sinergie Vol. 37, Issue 3, 2019

consumatori sostengono che acquisterebbero ugualmente il prodotto se percepiscono positivamente la sua immagine o, comunque, che difficilmente lo osteggerebbero (Carrigan e Attalla, 2001).

Ciò trova giustificazione, in primo luogo, nella tendenza degli individui a confermare le scelte adottate in passato, evitando o ridimensionando le informazioni potenzialmente destabilizzanti, al fine di non incorrere in stati di disequilibro o di dissonanza cognitiva (Heider, 1958; Festinger, 1957; Cooper, 2007). In secondo luogo, la componente fiduciaria della marca che in ultima analisi scaturisce dalla ripetuta conferma delle aspettative - genera nel consumatore una sorta di inerzia cognitiva, che, ancora una volta, riduce la sensibilità a nuove informazioni dissonanti rispetto a quelle già memorizzate. L'informazione sullaffidabilità della marca non avrebbe infatti alcun significato se il soggetto acquirente si proponesse di verificarla in ogni occasione di acquisto e consumo della marca stessa. Il cliente, per economizzare le proprie risorse cognitive e temporali, compie pertanto un atto di fiducia nella marca, confidando di ritrovare in tutte le sue manifestazioni le medesime valenze distintive (Vicari, 1995).

Questa inerzia cognitiva è maggiore per le associazioni più astratte, riguardanti la sfera valoriale del consumatore. La self knowledge, che secondo la teoria della catena mezzi-fini (Gutnam, 1982; Olson e Reynolds, 1983; Reynolds e Gutman, 2001; Manyiwa e Crawford, 2001; Orsingher et al., 2011) attiene al legame tra i benefici ricercati nella marca e i valori individuali, risulta più difficile da modificare rispetto alla product knowledge (cioè alla rappresentazione mentale delle caratteristiche del prodotto). La self knowledge, infatti, coinvolge maggiormente la componente affettiva degli atteggiamenti, che - essendo più legata alle funzioni di autodifesa e comunicativa da essi svolte nellambito del contesto psicologico e sociale dell'individuo (Katz, 1960) - manifesta una più elevata resistenza al cambiamento.

Pertanto, poiché lalterazione dei dati di product social responsibility coinvolge associazioni alla marca più astratte rispetto a quelle concernenti la product sophistication (che interessa la product knowledge), data la relazione positiva fra immagine di marca e intenzione di acquisto, è possibile avanzare le seguenti ipotesi:

H2) Una marca dotata di un'immagine forte, rispetto a una con un'immagine debole: a) evidenzia una maggiore intenzione di acquisto in presenza di entrambe le tipologie di alterazione dei dati; b) tale impatto è più elevato se lalterazione dei dati riguarda la product social responsibility.

Come anticipato nell'introduzione al lavoro, la letteratura evidenzia che le caratteristiche socio-demografiche non influiscono in misura rilevante sullintenzione d'acquisto del consumatore in presenza di comportamenti eticamente responsabili/irresponsabili dallazienda (De Pelsmacker et al., 2005). Diversamente avviene nel caso delle variabili psicografiche (Anderson e Cunningham, 1972; Cowe e Williams, 2000). A questo riguardo, vari autori (Dickson 2001; Huttel et al., 2018; Tey et al., 2018) hanno evidenziato l'importanza di variabili quali gli atteggiamenti e, soprattutto, i valori strumentali e terminali dell'individuo (Rokeach, 1973). 
Una variabile psicografica che rimane meritevole di approfondimento è rappresentata dalle caratteristiche della personalità, come ad esempio quelle che riflettono il "self concept" dell'individuo.

Tale costrutto è inteso quale complesso delle valutazioni e delle sensazioni relative a se stesso (Sirgy, 1982). Il self concept possiede una propria coerenza complessiva ed è relativamente stabile, quantomeno nel breve periodo. Esso rappresenta, pertanto, una fonte di inerzia e di rigidità nel comportamento dell'individuo, nella misura in cui questi si sforza di rendere coerente ciò che fa con ciò che pensa di sé. Inoltre, il self concept si caratterizza per una certa "autoreferenzialità", poiché l'individuo tende a interpretare gli stimoli ai quali è esposto coerentemente con le convinzioni e le valutazioni già maturate.

Gli studi sul tema hanno evidenziato l'esistenza e l'influenza indipendente di almeno due dimensioni di tale costrutto: coerenza con se stessi (selfconsistency) e autostima (selfesteem). La prima motiva l'individuo ad agire in modo coerente con la propria percezione di sé. La seconda, invece, alimenta il bisogno di orientare i comportamenti di consumo al perseguimento di obiettivi che permettano di mantenere o aumentare positivamente la propria self image (Sirgy, 1985). Quest'ultima dimensione è quella più rilevante ai nostri fini, in quanto è stato rilevato che il grado di autostima incide sulla sensibilità alle informazioni che orientano i processi decisionali dei consumatori. In particolare, gli individui con bassa autostima vengono maggiormente influenzati dalla comunicazione e risultano più sensibili ai messaggi negativi (Cohen, 1959; Leventhal e Perloe, 1962; Brockner, 1988; Campbell, 1990). Inoltre, come evidenziato da Rhodes e Wood (1992, pp. 158-159), "people high in self-esteem may use avoidance defense mechanisms, such as repression and reaction formation, which enable them to ignore or repress information threatening to their selfconcept. These types of defenses result in low levels of yielding".

Alla luce della correlazione negativa fra autostima e influenzabilità dell'individuo, si propone la terza ipotesi di ricerca:

H3) in presenza di unialterazione dei dati di product social responsibility, l'intenzione di acquisto del consumatore aumenta ulteriormente se a una forte brand image si associa unelevata autostima.

\section{La metodologia di ricerca}

La verifica delle ipotesi illustrate nelle pagine precedenti è avvenuta mediante un esperimento between subjects, nel quale gruppi diversi di individui sono stati casualmente esposti alle differenti condizioni sperimentali. L'esperimento si basa su un disegno fattoriale $2 \times 2 \times 2$, ossia su tre variabili indipendenti, ciascuna articolata su due livelli, e una variabile dipendente. Più precisamente, le variabili indipendenti sono rappresentate da: l'alterazione dei dati di prodotto (product sophistication vs product social responsibility), la brand image (forte $v s$ debole) e l'autostima dell'individuo (alta $v s$ bassa). La variabile dipendente è invece costituita dall'intenzione d'acquisto del consumatore.
Giuseppe Bertoli

Bruno Busacca

Alessandro Calzetti

La sensibilità del

consumatore a

comportamenti aziendali

eticamente scorretti 
sinergie Vol. 37, Issue 3, 2019

La figura 1 sintetizza il disegno sperimentale utilizzato. Come si nota, esso è composto da otto possibili condizioni, corrispondenti all'incrocio fra le tre variabili indipendenti e i loro rispettivi livelli. Le prime due variabili indipendenti (alterazione dei dati di prodotto e brand image) sono state proposte al campione coinvolto nella sperimentazione sotto forma di stimoli, mentre, come anticipato nell'introduzione, la terza variabile (autostima) è stata misurata nel corso dell'esperimento mediante la scala a 10 item proposta da Rosemberg (1965) e quindi distinta ex post in due livelli (alta $v s$ bassa) sulla base del valore mediano.

Fig. 1: Il disegno sperimentale

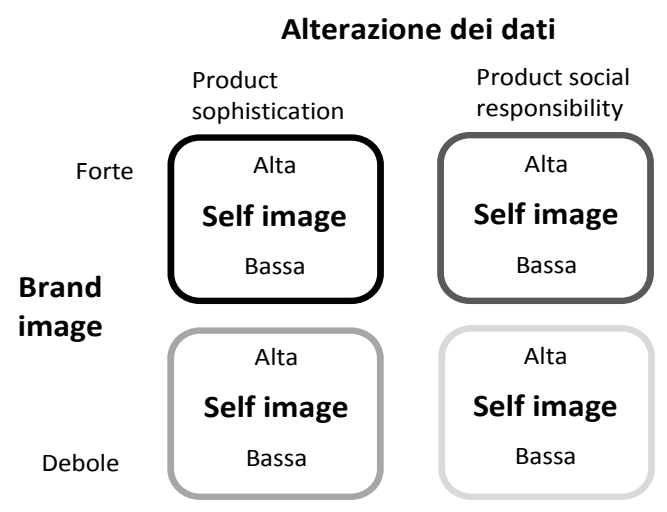

Fonte: elaborazione degli autori

Le quattro condizioni determinate dallincontro dei livelli dei due stimoli proposti sono state sottoposte a quattro gruppi differenti di soggetti. Successivamente, dopo aver misurato l'autostima individuale, si è proceduto a distinguere ulteriormente ciascuno di tali gruppi in due sottogruppi in funzione dei livelli assunti da tale variabile (alta $v s$ bassa), ottenendo così otto campioni differenti, uno per ciascuna delle condizioni previste dal disegno sperimentale.

La metodologia utilizzata per la realizzazione del disegno sperimentale si è articolata nelle seguenti fasi:

- definizione delle variabili oggetto di studio, scelta delle rispettive scale di misurazione e descrizione degli stimoli da sottoporre agli individui coinvolti nella sperimentazione;

- svolgimento di indagini preliminari finalizzate a verificare la corretta percezione degli stimoli proposti e l'adeguatezza del questionario da somministrare al campione indagato;

- selezione dei soggetti in riferimento ai quali svolgere la sperimentazione e svolgimento delle interviste.

\subsection{Le variabili oggetto di studio, le scale di misurazione e gli stimoli proposti}

Le variabili utilizzate nel disegno sperimentale sono state misurate ricorrendo a scale già testate nella letteratura relativa ai costrutti oggetto 
di analisi. Tutte le scale di misurazione utilizzate sono a 7 punti. Nello specifico, le variabili alterazione dei dati di prodotto, autostima e intenzione d'acquisto sono state misurate mediante una scala Likert, mentre la brand image con una scala bipolare. La tabella 1 evidenzia, per ciascuna variabile, gli item utilizzati, i coefficienti Alpha di Cronbach rilevati mediante i pretest e le fonti di riferimento.

Tab. 1: Le scale di misurazione utilizzate

\begin{tabular}{|l|c|l|c|}
\hline Variabili & $\begin{array}{c}\text { Numero } \\
\text { di item }\end{array}$ & \multicolumn{1}{|c|}{ Fonte } & $\begin{array}{c}\text { Alpha di } \\
\text { Cronbach }\end{array}$ \\
\hline Brand image dei dati di product & 3 & Brown e Dacin (1997) & .965 \\
\hline $\begin{array}{l}\text { Alterazione de } \\
\text { sophistication }\end{array}$ & 3 & Brown e Dacin (1997) & .945 \\
\hline $\begin{array}{l}\text { Alterazione dei dati di product social } \\
\text { responsibility }\end{array}$ & 3 & $\begin{array}{l}\text { Dodds, Monroe e e } \\
\text { Grewal (1991) }\end{array}$ & .963 \\
\hline Intenzione d'acquisto & 10 & Rosemberg (1965) & .889 \\
\hline Autostima & & \multicolumn{1}{|c|}{} \\
\hline
\end{tabular}

Gli stimoli sottoposti agli individui coinvolti nell'esperimento sono rappresentati dalle variabili indipendenti alterazione dei dati di prodotto e brand image. Nello specifico, sono stati presentati quattro scenari, derivanti dalla combinazione dei livelli dei suddetti stimoli e corrispondenti alle celle della matrice rappresentata nella precedente Figura 1.

Per quanto in particolare riguarda l'immagine di marca, al fine di innalzare il controllo sperimentale e meglio valutare le associazioni sviluppate dai rispondenti, la letteratura suggerisce l'utilizzo di brand name fittizi (Boush e Loken, 1991; Geuens e De Pelsmacker, 2017). Come nell'esperimento condotto da Brown e Dacin (1997, p. 72), è stata così utilizzata una marca ideata ad hoc, casualmente denominata "Rowfie" e collegata a un prodotto, altrettanto fittizio, indicato come "Nigiko3". Anche in questo caso, tale scelta è motivata dall'obiettivo di aumentare il controllo sperimentale, focalizzando l'attenzione degli intervistati solo sulle informazioni fornite nell'esperimento attraverso gli stimoli, evitando che il giudizio sul prodotto risulti influenzato dalla valutazione della categoria (Fiske e Pavelchak, 1986) e dal coinvolgimento psicologico verso la stessa (Petty et al., 2005).

La marca e il prodotto individuati sono stati presentati all'interno di un contesto "reale" (Low e Lamb, 2000): un articolo di giornale, il cui contenuto definisce quindi lo scenario proposto al rispondente.

Nel dettaglio, lo stimolo alterazione dei dati di prodotto è stato manipolato utilizzando le definizioni di product sophistication e di product social responsibility fornite dal citato studio di Brown e Dacin (1997) e sottoponendo agli intervistati, rispettivamente, le seguenti affermazioni:

- Rowfie è stata accusata di aver alterato i dati delle caratteristiche tecniche del suo ultimo prodotto "Nigiko3" e i test sul prodotto hanno confermato l'accusa. Nigiko3 non rispetta la qualità tecnica e nemmeno le prestazioni e l'innovatività promesse dall'azienda;

- Rowfie è stata accusata di aver alterato i dati di responsabilità sociale del
Giuseppe Bertoli

Bruno Busacca

Alessandro Calzetti La sensibilità del

consumatore a

comportamenti aziendali eticamente scorretti 
sinergie

Vol. 37, Issue 3, 2019 suo ultimo prodotto "Nigiko3" e i test sul prodotto hanno confermato l'accusa. Nigiko3 non rispetta l'ambiente e l'impegno sociale promesso dall'azienda.

Riguardo allo stimolo rappresentato dalla brand image, ai soggetti coinvolti nella sperimentazione è stata proposta una descrizione finalizzata a porre in evidenza le associazioni alla marca, con riferimento alla loro forza, positività e unicità (Keller, 2012). Più precisamente, la valenza dell'immagine è stata manipolata mediante le seguenti affermazioni polari in grado di rappresentare i suoi due livelli (forte $v s$ debole):

- Rowfie è unazienda di fama mondiale e si contraddistingue dai concorrenti per l'unicità dei suoi prodotti, occupando così i primi posti nella lista delle migliori aziende;

- Rowfie è unazienda poco conosciuta e non si contraddistingue dai concorrenti per l'unicità dei suoi prodotti, non figurando così ai primi posti nella lista delle migliori aziende.

\subsection{Le indagini preliminari}

Al fine di verificare la corretta percezione degli stimoli proposti e sviluppare al meglio il questionario da sottoporre ai soggetti coinvolti nella sperimentazione, è stato svolto un pre-test.

A un campione di convenienza composto da venti rispondenti di origine italiana (60\% maschi e $40 \%$ femmine), con età media di 24 anni e con un elevato livello di istruzione (90\% tra studenti universitari e studenti lavoratori e $10 \%$ lavoratori a tempo pieno con precedente percorso universitario), è stato somministrato in maniera casuale un questionario. A ciascun partecipante è stata sottoposta una delle quattro condizioni sperimentali rappresentate nella precedente Figura 1, in modo da ottenere un numero uguale di risposte per ciascuna condizione.

Nel pre-test, per entrambe le variabili indicate sugli assi di tale figura, è stata effettuata unanalisi T-test su due campioni indipendenti. Per quanto riguarda la variabile brand image, i rispondenti hanno valutato lo stimolo sulla base della scala di misurazione proposta da Low e Lamb (2000). Il risultato del T-test ha rilevato che la percezione dello stimolo brand image forte $(\mathrm{M}=4.44) ; \mathrm{SD}=.55)$ è significativamente diversa $(\mathrm{t}(18)=7.53 ; \mathrm{p}<.001)$ da quella di brand image debole $(\mathrm{M}=2.76 ; \mathrm{SD}=.43)$, confermando così ladeguata comprensione dello stimolo proposto.

In relazione alla variabile alterazione dei dati di prodotto, i rispondenti hanno valutato lo stimolo sulla base della scala di misurazione proposta da Brown e Dacin (1997). Attenendo tale alterazione a una problematica etica e assumendo quindi, per sua natura, valenza negativa per entrambi i suoi livelli (product sophistication e produc social responsbility), si è condotta unanalisi T-test per verificare la distanza di ciascun livello della variabile indipendente dalla valutazione complessiva del prodotto. I risultati del test hanno confermato, per entrambi i livelli, la corretta percezione dello stimolo in questione, individuando conseguentemente una distanza significativa delle caratteristiche di prodotto alterate dalla valutazione globale del prodotto. Nello specifico, la percezione del livello product sophistication $(\mathrm{M}=1,70 ; \mathrm{SD}=.43)$ è risultata significativamente diversa 
$(\mathrm{t}(18)=3.75 ; \mathrm{p}<.005)$ dalla valutazione complessiva del prodotto $(\mathrm{M}=3.10$; $\mathrm{SD}=1.10)$. Allo stesso modo, la percezione del livello product social responsibility $(\mathrm{M}=1.36 ; \mathrm{SD}=.37)$ è significativamente diversa $(\mathrm{t}(18)=5.70$; $\mathrm{p}<.001)$ da tale valutazione $(\mathrm{M}=4.10 ; \mathrm{SD}=1.37)$.

Infine, è stata valutata l'adeguatezza del testo del questionario da somministrare al campione di soggetti coinvolti nell'indagine, chiedendo ai partecipanti al pre-test di valutare la chiarezza dei quesiti proposti. In base alle loro osservazioni, si è proceduto alla modifica del testo concernente gli stimoli individuati e alla formulazione di alcune domande.

Il questionario somministrato è suddiviso in cinque sezioni:

- introduzione, nella quale viene presentato il tema generale della ricerca, la durata stimata dell'impegno richiesto al rispondente e la garanzia per il rispetto della privacy;

- breve descrizione dell'azienda fittizia Rowfie, al fine di generare un'idea relativamente alla stessa e rendere più veritiero l'esperimento (Kanar et al., 2010). Ai partecipanti è stato comunicato che "Rowfie e un'azienda diversificata operante in vari settori (informatica, telecomunicazioni, sanità, bancario, industriale, immobiliare, distribuzione al dettaglio). Rowfie produce sia prodotti per l'industria sia beni di consumo e servizi destinati ai consumatori finali. L'ultimo prodotto di Rowfie è il dispositivo Nigiko3";

- presentazione degli stimoli dellesperimento, sottoponendo ai rispondenti, in maniera casuale e all'interno di un ipotetico articolo di giornale contenente le informazioni indicate nel paragrafo precedente, una delle quattro condizioni rappresentate della precedente Figura 1 e determinate dall'incontro dei livelli dei due stimoli considerati, in modo da ricevere il medesimo numero di risposte riguardo a ciascuna di tali condizioni;

- misurazione delle variabili oggetto di studio (alterazione dei dati di prodotto, brand image, autostima, intenzione d'acquisto) con le rispettive scale;

- domande sulle caratteristiche socio-demografiche dei rispondenti.

\subsection{Il campione}

Il questionario è stato somministrato a un campione formato da 260 soggetti di origine italiana, di età compresa fra i 18 e i 60 anni. Il campione è composto prevalentemente da lavoratori e da studenti universitari, provenienti principalmente da corsi di studio di tipo economico e ingegneristico attivati dalle università milanesi e da business school straniere. Il metodo di campionamento utilizzato è quello non probabilistico per convenienza. Il questionario è stato distribuito sia online che di persona con il supporto di dispositivi digitali.

Sono state ottenute 218 risposte. Depurando i questionari incompleti, è stato possibile selezionare 200 osservazioni valide, ossia 50 per ciascuna delle quattro celle sperimentali indicate nella precedente Figura 1. Il 55\% dei rispondenti è di sesso maschile, il $58 \%$ proviene dal nord Italia $(20 \%$ dal centro e $22 \%$ dal sud). L’età media è pari a 32,9 anni: il $61 \%$ ha un'età compresa fra i 18 e i 30 anni, l' $11 \%$ fra 31 e 40 e il $17 \%$ tra i 50 e i 60
Giuseppe Bertoli

Bruno Busacca

Alessandro Calzetti

La sensibilità del

consumatore a

comportamenti aziendali eticamente scorretti 
sinergie Vol. 37, Issue 3, 2019

anni. Il campione è caratterizzato da un elevato livello di istruzione: il $18 \%$ possiede il diploma di maturità, il $40 \%$ la laurea triennale e il $42 \%$ la laurea magistrale o a ciclo unico. Per quanto riguarda l'occupazione, il 52\% dei rispondenti sono lavoratori a tempo pieno, il 6\% lavoratori a tempo parziale, il 2\% persone in cerca di occupazione, mentre gli studenti e gli studenti lavoratori rappresentano ciascuno il $20 \%$ del totale.

\section{I risultati della sperimentazione}

La nostra ricerca ipotizza, in primo luogo, che l'alterazione dei dati di product sophistication da parte di un'impresa influenzi in misura maggiormente negativa l'intenzione d'acquisto del consumatore rispetto allalterazione dei dati di product social responsibility. Per la verifica di tale ipotesi, si è condotta unanalisi T-test su due gruppi, ciascuno composto da 100 individui esposti a una delle due tipologie di alterazione dei dati. Si è quindi proceduto al confronto dell'intenzione media di acquisto espressa dai due gruppi.

I risultati dell'analisi (Figura 2) evidenziano chela media delle valutazioni di tale intenzione è significativamente diversa $(\mathrm{p}<.001)$. In particolare, l'intenzione media di acquisto in situazioni di alterazione dei dati di product sophistication $(\mathrm{M}=1.93 ; \mathrm{SD}=.93)$ è risultata significativamente inferiore $(\mathrm{t}(151.6)=-7.32 ; \mathrm{p}<.001)$ rispetto a quella rilevata allorché l'alterazione riguarda i dati di product social responsibility $(\mathrm{M}=3.38 ; \mathrm{SD}=1.74)$. L'ipotesi H1 risulta quindi confermata.

Fig. 2: Confronto delle medie dell'intenzione di acquisto per $i$ livelli product sophistication e product social responsibility della variabile indipendente alterazione dei dati di prodotto

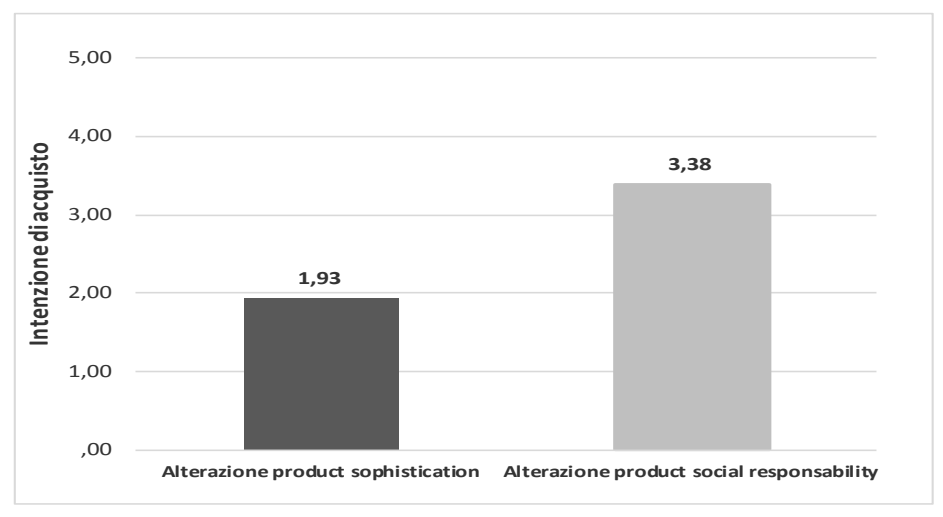

Fonte: elaborazione degli autori

La seconda ipotesi di ricerca oggetto di verifica sostiene che, in presenza di una marca dotata di un'immagine forte, l'intenzione di acquisto del consumatore è più elevata se l'alterazione dei dati riguarda la product social responsibility anziché la product sophistication. Al fine 
di verificare tale ipotesi, si è condotta un'analisi della varianza fattoriale univariata. Dall'output di tale analisi (Tabella 2), si nota che tanto le singole variabili brand image $(\mathrm{p}<.001)$ e alterazione dei dati di prodotto $(\mathrm{p}<.001)$ quanto la loro interazione $(\mathrm{p}<.001)$ influenzano in misura statisticamente significativa l'intenzione di acquisto.

Tab. 2: Risultati dellanalisi della varianza fattoriale univariata

\begin{tabular}{|l|l|l|l|l|l|}
\hline Source & $\begin{array}{c}\text { Type III Sum } \\
\text { of Squares }\end{array}$ & Df & Mean Square & \multicolumn{1}{|c|}{ F } & Sig. \\
\hline Corrected Model & $284,175^{\text {a }}$ & 3 & 94,725 & 88,815 &, 000 \\
\hline Intercept & 1416,894 & 1 & 1416,894 & 1328,493 &, 000 \\
\hline Brand image & 139,445 & 1 & 139,445 & 130,745 &, 000 \\
\hline Alterazione & 105,125 & 1 & 105,125 & 98,566 &, 000 \\
\hline Brand image * Alterazione & 39,605 & 1 & 39,605 & 37,134 &, 000 \\
\hline Error & 209,042 & 196 & 1,067 & & \\
\hline Total & 1910,111 & 200 & & & \\
\hline Corrected Total & 493,217 & 199 & & & \\
\hline
\end{tabular}

a. R Squared $=, 576$ (Adjusted R Squared $=, 570$ )

Fonte: elaborazione degli autori.

La Figura 3 permette di verificare l'ipotesi H2. Come si nota, l'immagine di marca svolge un ruolo "protettivo" in presenza di entrambe le tipologie di alterazione dei dati esaminate $(\mathrm{H} 2 \mathrm{a})$, ma tale protezione è significativamente più elevata laddove l'alterazione riguardi la product social responsibility $(\mathrm{H} 2 \mathrm{~b})$. Infatti, per una marca dotata di un'immagine forte, rispetto a una con un'immagine debole, l'intenzione di acquisto aumenta:

- del 50,6\% (da 1,54 a 2,32), in caso di alterazione dei dati di product sophistication;

- del 122\% (da 2,10 a 4,66), in presenza di alterazione dei dati di product social responsibility.

Fig. 3: Effetto delle variabili indipendenti brand image e alterazione dei dati sulla variabile dipendente intenzione di acquisto

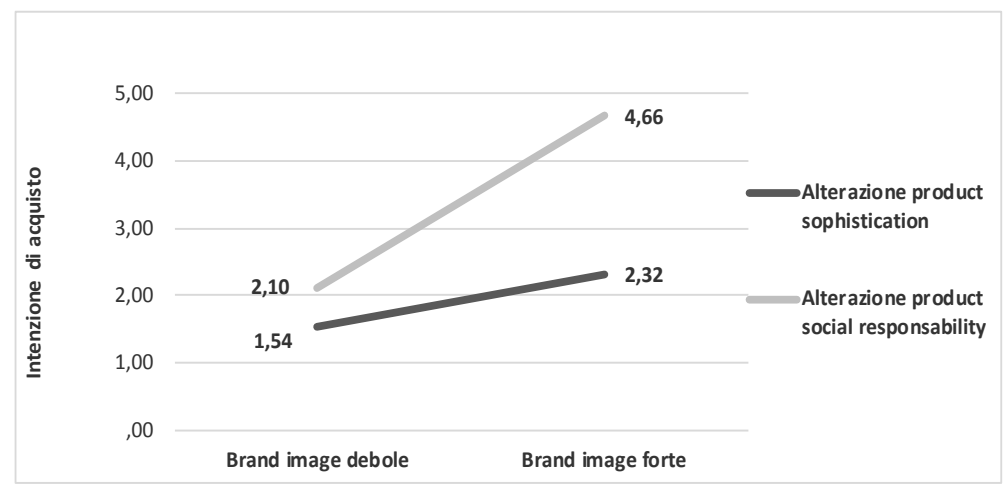

Fonte: elaborazione degli autori.
Giuseppe Bertoli

Bruno Busacca

Alessandro Calzetti

La sensibilità del

consumatore a

comportamenti aziendali

eticamente scorretti 
sinergie

Vol. 37, Issue 3, 2019

Con la terza ipotesi di ricerca viene introdotta la variabile indipendente autostima. Come anticipato, tale variabile, a differenza degli stimoli sin qui considerati (brand image e alterazione dei dati), è stata valutata ex-post e distinta in due livelli (alta $v s$ bassa) rispetto al valore assunto dalla mediana. Si è quindi condotta unanalisi della varianza fattoriale univariata al fine di valutare l'effetto delle tre variabili indipendenti (brand image, alterazione dei dati di prodotto e autostima) sulla variabile dipendente (intenzione di acquisto).

Dai risultati dell'analisi (Tabella 3), si evince che le singole variabili brand image $(\mathrm{p}<.001)$ e alterazione dei dati di prodotto $(\mathrm{p}<.001)$ esercitano un effetto significativo sullintenzione di acquisto, mentre l'impatto dellautostima non risulta avere rilevanza statistica $(\mathrm{p}=.081)$. Tutte le interazioni delle variabili indipendenti a due livelli hanno un effetto significativo sulla variabile dipendente $(\mathrm{p}<.001)$. È anche statisticamente significativo l'effetto dell'interazione fra brand image, alterazione dei dati di prodotto e autostima $(\mathrm{p}=.003)$.

Tab. 3: Output dell'analisi della varianza fattoriale univariata (Tests of BetweenSubjects Effects - Dependent Variable: Intenzione d'acquisto)

\begin{tabular}{|l|l|l|l|l|l|}
\hline Source & $\begin{array}{l}\text { Type III Sum } \\
\text { of Squares }\end{array}$ & Df & Mean Square & \multicolumn{1}{|c|}{ F } & Sig. \\
\hline Corrected Model & $344,259 \mathrm{a}$ & 7 & 49,180 & 63,391 &, 000 \\
\hline Intercept & 1380,203 & 1 & 1380,203 & 1779,019 &, 000 \\
\hline Brand image & 129,790 & 1 & 129,790 & 167,293 &, 000 \\
\hline Alterazione & 91,703 & 1 & 91,703 & 118,201 &, 000 \\
\hline Autostima & 2,384 & 1 & 2,384 & 3,073 &, 081 \\
\hline Brand image ${ }^{*}$ Alterazione & 33,504 & 1 & 33,504 & 43,186 &, 000 \\
\hline Brand image ${ }^{*}$ Autostima & 16,070 & 1 & 16,070 & 20,714 &, 000 \\
\hline Alterazione * Autostima & 34,845 & 1 & 34,845 & 44,913 &, 000 \\
\hline Brand image* Alterazione ${ }^{*}$ Autostima & 6,843 & 1 & 6,843 & 8,820 &, 003 \\
\hline Error & 148,958 & 192 &, 776 & & \\
\hline Total & 1910,111 & 200 & & & \\
\hline Corrected Total & 493,217 & 199 & & & \\
\hline
\end{tabular}

a. R Squared =,698 (Adjusted R Squared =,687)

Fonte: elaborazione degli autori.

A conferma dell'ipotesi $\mathrm{H} 3$, la media delle valutazioni dell'intenzione di acquisto per una marca con immagine forte che abbia alterato i dati di product social responsibility è significativamente più elevata per individui con alta autostima $(\mathrm{M}=5.54 ; \mathrm{SD}=.42)$ rispetto a quella espressa da soggetti con autostima bassa $(\mathrm{M}=3.54 ; \mathrm{SD}=1.67)$. Peraltro, l'intenzione media di acquisto pari a 5,54 è significativamente superiore rispetto a quella rilevata in tutte le altre combinazioni dei livelli delle tre variabili indipendenti (tabella 4). Di conseguenza, si può confermare che, in presenza di un'alterazione dei dati di product social responsibility, l'intenzione di acquisto del consumatore si accresce ulteriormente se a unelevata brand image si associa un'alta autostima. 
Tab. 4: Statistiche descrittive dell'intenzione di acquisto nelle combinazioni dei livelli delle tre variabili indipendenti

\begin{tabular}{|l|l|l|l|l|l|}
\hline \multirow{2}{*}{ Brand image } & Alterazione & Autostima & Mean & \multicolumn{1}{c|}{$\begin{array}{c}\text { Std. } \\
\text { Deviation }\end{array}$} & N \\
\hline \multirow{3}{*}{ BI_Debole } & \multirow{2}{*}{ ALT_Product sophistication } & Bassa & 1,9722 &, 95258 & 24 \\
\cline { 3 - 6 } & & Alta & 1,1538 &, 23534 & 26 \\
\cline { 3 - 6 } & \multirow{2}{*}{ ALT_Product social responsibility } & Bassa & 2,0435 &, 92826 & 23 \\
\cline { 3 - 6 } & & Alta & 2,1605 &, 42738 & 27 \\
\hline \multirow{2}{*}{ BI_Forte } & \multirow{2}{*}{ ALT_Product sophistication } & Bassa & 2,5714 & 1,17446 & 21 \\
\cline { 3 - 6 } & & Alta & 2,1494 &, 63362 & 29 \\
\cline { 3 - 6 } & \multirow{2}{*}{ ALT_Product social responsibility } & Bassa & 3,5455 & 1,67946 & 22 \\
\cline { 3 - 6 } & & Alta & 5,5476 &, 42759 & 28 \\
\hline
\end{tabular}

Fonte: elaborazioni degli autori.

\section{Implicazioni teoriche e manageriali}

Le analisi della varianza effettuate hanno confermato le ipotesi di ricerca formulate. In particolare, il consumatore è influenzato in misura maggiormente negativa dall'alterazione dei dati di product sophistication che non da quella concernente la product social responsibility. Ciò dimostra che le decisioni del consumatore sono maggiormente influenzate dalla qualità e dalla funzionalità del prodotto che non dalla sua conformità alle "societal obbligation" dell'impresa. Se ne deduce che, quantunque sia le associazioni di product sophistication sia quelle di product social responsibility siano importanti ai fini della reputazione aziendale, per il consumatore è più rilevante che il prodotto rispetti la qualità e le performance dichiarate dall'azienda.

È inoltre possibile affermare che, per entrambe le tipologie di alterazione dei dati considerate, l'immagine di marca esercita un forte impatto sull'intenzione d'acquisto. Infatti, in presenza di una forte brand image, il consumatore è più propenso ad acquistare il prodotto a prescindere dal tipo di dati alterati.

Alla luce della positiva verifica dell'ipotesi $H 2$, un risultato particolarmente interessante per le strategie aziendali è rappresentato dall'effetto dell'interazione dell'immagine di marca con l'alterazione dei dati di prodotto. In particolare, se l'alterazione riguarda i dati di product social responsibility ed è compiuta da un'impresa che dispone di un'elevata brand image, si riscontra un'intenzione d'acquisto superiore a quella rilevata in tutte le altre condizioni. In coerenza, quindi, con quanto riscontrato a proposito dell'ipotesi $H 1$, l'alterazione dei dati relativi all'attività sociale e ambientale ha un impatto minore sulle decisioni del consumatore, in particolare quando l'impresa ha saputo sviluppare associazioni forti, favorevoli e uniche alla propria marca. Al contrario, l'alterazione dei dati di product sophistication, soprattutto in presenza di brand image debole, comporta l'intenzione media di acquisto più bassa. È fondamentale, quindi, che il management aziendale sia in grado di costruire e rafforzare nel tempo l'immagine di marca. Le associazioni al brand, infatti, orientano le aspettative e le percezioni del consumatore verso i benefici funzionali,
Giuseppe Bertoli Bruno Busacca Alessandro Calzetti La sensibilità del consumatore a

comportamenti aziendali eticamente scorretti . 
sinergie

Vol. 37, Issue 3, 2019

simbolici ed esperienziali dell'offerta, alimentando un capitale di fiducia in grado di proteggere l'azienda dall'impatto di informazioni destabilizzanti.

Da qui alcune fondamentali implicazioni, nella prospettiva dell'impresa e in quella dell'interesse collettivo.

Per quanto riguarda l'impresa, pare evidente che il management debba riservare grande attenzione:

- alla verifica dell'attendibilità delle informazioni comunicate al mercato relativamente agli attributi, alla qualità e alla performance dei prodotti. Ciò anche in considerazione della crescente diffusione delle recensioni su tali caratteristiche spontaneamente pubblicate dai consumatori negli ambienti digitali e della spirale di passaparola negativo che si innescherebbe ove gli utilizzatori rilevassero che le caratteristiche dichiarate dall'azienda divergono da quelle reali (Tassiello et al., 2017; Amatulli et al., 2018);

- alla costruzione e comunicazione dell'immagine della marca. L'effetto di una brand image forte sull'intenzione d'acquisto in presenza di entrambi i tipi di alterazione dei dati di prodotto conferma l'importanza strategica di questa risorsa immateriale, in grado di attutire significativamente l'impatto del comportamento eticamente scorretto posto in essere da un'impresa. I consumatori, infatti, tendono a non venire influenzati da informazioni negative concernenti marche affermate e a selezionare le informazioni coerentemente con le loro opinioni (Ahluwalia, 2002).

Le suddette riflessioni assumono tuttavia un'implicazione che chiama in causa l'interesse collettivo: il minore impatto dell'alterazione legata alla responsabilità sociale sulle scelte del consumatore, ulteriormente attenuato dallo "scudo" rappresentato dall'immagine di marca, evidenzia la parziale inefficacia del mercato nel sanzionare comportamenti eticamente scorretti. E pertanto doveroso trovare un rimedio a tale inefficacia, elevando in primis la sensibilità del consumatore a tali tematiche. Il punto verrà ripreso nelle considerazioni conclusive.

Con la terza ipotesi di ricerca è stata introdotta la variabile autostima che, rappresentando una componente centrale del self concept dell'individuo, ne influenza la sensibilità alle informazioni che orientano i suoi processi decisionali. I dati rilevati dimostrano che l'autostima, singolarmente considerata, pur non esercitando un impatto statisticamente rilevante sull'intenzione d'acquisto del consumatore, manifesta un effetto di interazione significativo con le altre variabili indipendenti considerate nell'analisi.

In particolare, in presenza di alterazione di dati relativi alla responsabilità sociale, se a una forte immagine di marca si associa unelevata autostima, l'intenzione di acquisto del consumatore assume il valore più elevato. Per le marche caratterizzate da associazioni forti, favorevoli e uniche si rivela quindi opportuna la segmentazione dei consumatori sulla base del grado di autostima, per identificare il cluster meno influenzabile dall'insorgere di informazioni negative sui prodotti dell'impresa. È peraltro interessante sottolineare che tale opportunità permane anche nel caso di alterazione dei dati di product sophistication, dove l'intenzione di acquisto si rivela invece decrescente. In altre parole, anche se la maggiore sensibilità del consumatore all'alterazione dei dati di prodotto impatta negativamente 
sull'intenzione di acquisto, l'interazione fra la forza dell'immagine di marca e l'elevata autostima dell'individuo determina una minore riduzione della suddetta intenzione (Figura 4).

Fig. 4: Effetto delle variabili indipendenti brand image e autostima sulla variabile dipendente intenzione di acquisto, nelle due tipologie di alterazione dei dati

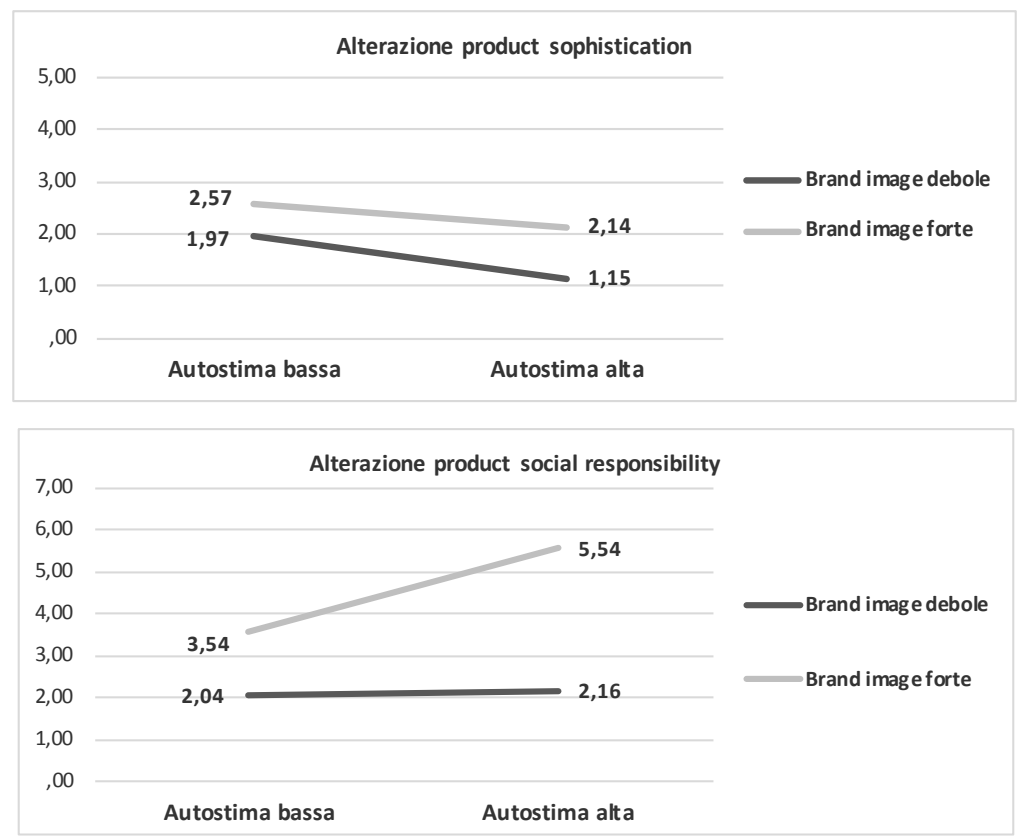

Fonte: elaborazioni degli autori

\section{Considerazioni finali}

La letteratura ha studiato negli anni l'importanza dell'etica aziendale per il consumatore, l'impatto (asimmetrico) dei comportamenti corretti e scorretti sull'immagine dell'impresa, le implicazioni connesse alla vicinanza del core business aziendale alle tematiche di CSR, all'immagine della sua marca $\mathrm{o}$, ancora, alla superiorità degli attributi di prodotto. Alcuni studi recenti si sono occupati di investigare se il comportamento eticamente scorretto di un'impresa, ad esempio l'alterazione dei dati di un prodotto, possa riflettersi negativamente sull'intera categoria merceologica (Trump e Newman, 2017).

Con riferimento ai filoni di indagine alla base delle ipotesi di ricerca, il nostro studio conferma l'utilità della distinzione fra associazioni cognitive legate alle abilità dell'impresa e alla sua responsabilità sociale ai fini della valutazione delle risposte dei consumatori a comportamenti aziendali non etici. Conferma inoltre che su tali risposte possono esercitare un effetto rilevante le variabili psicologiche individuali, introducendo un costrutto (l'autostima) che non ci risulta essere stato indagato nell'ambito di ricerca
Giuseppe Bertoli

Bruno Busacca

Alessandro Calzetti

La sensibilità del

consumatore a

comportamenti aziendali eticamente scorretti 
sinergie Vol. 37, Issue 3, 2019

qui considerato. Inoltre, i risultati emersi dallesperimento descritto nelle pagine precedenti forniscono un'ulteriore evidenza dell'effetto protettivo derivante da una forte brand image (Sen e Bhattacharya, 2001), contribuendo in modo originale a chiarire l'effetto di interazione fra immagine di marca, tipologia di alterazione dei dati e autostima dell'individuo.

In sintesi, i risultati delle analisi svolte hanno dimostrato che l'alterazione dei dati relativi alla qualità e alla funzionalità del prodotto influenza in misura maggiormente negativa l'intenzione d'acquisto del consumatore rispetto a quella concernente i dati di responsabilità sociale e che un'immagine di marca forte attutisce i comportamenti scorretti delle imprese, in particolare quando l'alterazione concerne tale seconda tipologia di dati e gli individui presentano elevati livelli di autostima.

Alla luce delle variabili in grado di limitare la sensibilità dei consumatori ai comportamenti socialmente responsabili delle aziende, un tema meritevole di attenta riflessione e di futuri approfondimenti, al quale sembra opportuno accennare in chiusura del lavoro, è rappresentato dalle iniziative atte $\mathrm{a}$ far sì che, per il benessere della collettività, tale sensibilità si diffonda, stimolando così le imprese all'adozione di comportamenti virtuosi (Du et al., 2010).

In linea di principio, queste iniziative potrebbero essere adottate dal management, il quale tuttavia non sempre è adeguatamente incentivato a investire in azioni di CRS, dal momento che i ritorni economico-finanziari di queste ultime potrebbero essere controversi (Barnett, 2007; Perrini et al., 2011). Un'altra possibilità è quella di far leva sulle organizzazioni non-profit, le quali possono amplificare le attività di CSR e porre in evidenza gli eventuali comportamenti scorretti delle imprese. A questo riguardo è tuttavia essenziale che, successivamente alla segnalazione di tali comportamenti, vengano posti in essere opportuni interventi sanzionatori. Unulteriore soluzione è rappresentata dall'adozione, da parte delle istituzioni nazionali o sovranazionali, di una regolamentazione finalizzata a incoraggiare e a sostenere l'adozione di comportamenti eticamente corretti da parte delle imprese, stimolando per tale via il loro contributo al benessere sociale.

Naturalmente, il presente lavoro non è esente da limiti, che potrebbero essererimossida eventualiricerchefuture.In primoluogo, lageneralizzabilità dei risultati è limitata dalla numerosità e dalla composizione del campione di soggetti coinvolto nellesperimento. In secondo luogo, non è stato considerato il livello di coinvolgimento psicologico del consumatore nei confronti delle tematiche etiche in generale e di alcune di esse in particolare. In terzo luogo, se da un lato l'utilizzo di una marca e di un prodotto fittizi consente un maggiore controllo sperimentale, esso impedisce dall'altro di considerare i legami che gli individui sviluppano con $\mathrm{i}$ brand reali. Ciò non permette di valutare l'impatto della fedeltà alla marca e della percezione della sua unicità da parte dei consumatori, né di testare l'effetto di possibili variabili di mediazione e/o di moderazione (come ad esempio l'intensità degli investimenti in comunicazione o il livello di product involvement). Inoltre, nell'ambito delle variabili psicografiche suscettibili di influenzare le scelte del consumatore in presenza di comportamenti aziendali eticamente irresponsabili, si è concentrata l'attenzione solo su una dimensione del 
self concept dell'individuo. Ricerche future potrebbero sia indagare altre variabili psicografiche sia approfondire l'impatto della self consistency. Infine, lesperimento è stato condotto con riferimento a un campione di consumatori solo italiani. Potrebbe pertanto essere interessante considerare l'impatto della diversità culturale, coinvolgendo nella sperimentazione individui appartenenti a paesi diversi sotto il profilo della sensibilità nei confronti di tematiche etiche.

\section{Bibliografia}

AAKER D.A. (1995), Building strong brands, The Free Press, New York.

AHLUWALIA R. (2002), "How prevalent is the negativity effect in consumer environments?", Journal of Consumer Research, vol. 29, n. 2, pp. 270-279.

AMATUlLI C., DE ANGELIS M., TASSIELlO V. (2018), "Efficacia delle raccomandazioni online relative ai servizi”, Micro\&Macro Marketing, n. 1, pp. 39-56.

ANDERSON T.W., CUNNINGHAM W.H. (1972), "The socially conscious consumer", Journal of Marketing, vol. 36, summer, pp. 23-31.

AUGER P., BURKE P., DEVINNEY T.M., LOUVIERE J.J. (2003), "What will consumers pay for social product features", Journal of Business Ethics, vol. 42, n. 3, pp. 281-304.

BARNETT M.L. (2007), "Stakeholder influence capacity and the variability of financial returns to corporate sociale responsibility", Academy of Management Review, vol. 32, n. 3, pp. 794-816,

BERENS G., van RIEL C.B.M., van REKOM J. (2007), “The CSR-Quality trade off: when can corporate social responsibility and corporate ability compensate each others?", Journal of Business Ethics, vol. 74, n. 3, pp. 233-52.

BOUSH D.M., LOKEN B. (1991), "A process-tracing study of brand extension evaluation", Journal of Marketing Research, vol. 28, n. 1, pp. 16-28.

BROCKNER J. (1988), Self-Esteem at Work: Research, Theory and Practice, D.C. Heath \& Co, Lexington.

BROWN T.J., DACIN P.A. (1997), "The company and the product: corporate associations and consumer Product response", Journal of Marketing, vol. 61, n. 1, pp. 68-84.

CAMPBELL J.P. (1990), "Modeling the performance prediction problem in industrial and organizational psychology", in Dunnette M.D., Hough L.M. (Eds.), Handbook of Industrial and Organizational Psychology, Consulting Psychologists Press, Inc, Palo Alto, CA, pp. 687-732.

CARRIGAN M., ATTALLA A. (2001), "The myth of the ethical consumer. Do ethics matter in purchase behavior?", Journal of Consumer Marketing, vol. 18, n. 7, pp. 560-77.

COHEN A.R. (1959), "Some implications of self-esteem for social influence”, in Janis L. et al. (eds.), Personality and Persuasibility, Yale University Press, New Haven CT, pp. 102-20.

COOPER J. (2007), Cognitive Dissonance: Fifty Years of a Classic Theory, SAGE Publications, Los Angeles.

COWE R., WILLIAMS S. (2000), Who Are the Ethical Consumers?, Booklet for the Co-operative Bank, Manchester.
Giuseppe Bertoli

Bruno Busacca

Alessandro Calzetti

La sensibilità del

consumatore a

comportamenti aziendali

eticamente scorretti 
DE PELSMACKER P., DRIESEN L., RAYP G. (2005), "Do consumers care about ethics? Willingness to pay for fair-trade coffee", The Journal of Consumer Affairs, vol. 39, n. 2, pp. 363-85.

DICKSON M.A. (2000), "Personal values, beliefs, knowledge, and attitudes relating to intentions to purchase Aapparel from socially responsible businesses", Clothing and Textiles Research Journal, vol. 18, n. 1, pp. 19-30.

DICKSON M.A. (2001), "Utility of no sweat labels for apparel consumers: profiling label users and predicting their purchases", The Journal of Consumer Affairs, vol. 35, n. 1, pp. 96-119.

DODDS W.B., MONROE K.B., GREWAL D. (1991), "Effects of price, brand and store information on buyers' product evaluations", Journal of Marketing Research, vol. 28, n. 3, pp. 307-19.

DU S., BHATTACHARYA C.B., SEN S. (2010), "Maximizing business returns to corporate social responsibility (CSR): The role of CSR communication", International Journal of Management Reviews, vol. 12, n. 1, pp. 8-19.

FESTINGER L.A. (1957), "A Theory of Cognitive Dissonance”, California, Stanford University Press 1957 (tr. it. Teoria della dissonanza cognitiva, Angeli, Milano, 1973).

FERREL O.C., GRESHAM L.G. (1985), “A contingency framework for understanding ethical decision making in marketing", Journal of Marketing, vol. 49, n. 3, pp. 87-96.

FISKE S.T., PAVELCHAK M.A. (1986), "Category-based versus piecemealbased affective responses: Developments in schema-triggered affect", in Sorrentino R.M., Higgins E.T., The Handbook of Motivation and Cognition, vol. 1: Foundations of Social Behavior, New York: Guilford, pp. 167-203.

FOLKES V.S., KAMINS M.A. (1999), "Effects of information about firms' ethical and unethical actions on consumers' Attitudes”, Journal of Consumer Psychology, vol. 8, pp. 243-259.

GEUENS M., DE PELSMACKER P. (2017), "Planning and conducting experimental advertising research and questionnaire design”, Journal of Advertising, vol. 46, n. 1, pp. 83-100.

GUTMAN J. (1982), "A means-end chain model based on consumer categorization processes", Journal of Marketing, vol. 46, n. 2, spring, pp. 60-72.

HEIDER F. (1958), The Psychology of Interpersonal Relations, John Wiley \& Sons, New York.

HUNT S.D., CHONKO L.B. (1985), "Ethics and marketing management: an empirical examination”, Journal of Business Research, vol. 23, n. 4, pp. 339-59.

HUNT S.D., CHONKO L.B., WILCOX J.B. (1984), "Ethical problems of marketing researchers", Journal of Marketing Research, vol. 21, n. 3, pp. 309-24.

HUTTEL A., ZIESEMER F., PEYER M., BALDERJAHN I. (2018), “To purchase or not? Why consumers make economically (non-) sustainable consumption choices", Journal of Cleaner Production, vol. 174, pp. 827-36.

KANAR A.D., COLLINS C.J., BELL B.S. (2009), "A comparison of the effects of positive and negative information on job seekers' organizational attraction and attribute recall", Human Performance, vol. 23, n. 3, pp. 193-212.

KATZ D. (1960), "The functional approach to the study of attitudes", Public Opinion Quarterly, vol. 24, n. 2, pp. 163-204.

KELLER K.L. (2012), Strategic Brand Management, Upper Saddle River, NJ: Prentice-Hall, 4th edition 
KELLER K.L., AAKER D.A. (1992), "The effects of sequential introduction of Giuseppe Bertoli brand extensions", Journal of Marketing Research, vol. 29, n. 1, pp. 35-50. $\quad \begin{aligned} & \text { Blessandro Calzetti } \\ & \text { Alestions }\end{aligned}$

JOHNSON Z.S., LEE Y.J., ASHOORI M.T. (2018), "Brand associations: the value of La sensibilità del ability versus social responsibility depends on consumer goals", Journal of comportamenti aziendali Brand Management, vol. 25, n. 1, pp. 27-37.

LEVENTHAL H., PERLOE S.I. (1962), "A relationship between self-esteem and persuasibility", Journal of Abnormal and Social Psychology, vol. 64, n. 5, pp. 385-88.

LOW G.S., LAMB Jr. C.W. (2000), “The measurement and dimensionality of brand associations", Journal of Product and Brand Management, vol. 9, n. 6, pp. 350-68.

MANYIWA S., CRAWFORD I. (2001), "Determining linkages between consumer choices in a social context and the consumer's values: A means-end approach", Journal of Consumer Behavior, vol. 2, n. 1, pp. 54-70.

MOHR L.A., WEBB D.J. (2005), “The Effects of corporate social responsibility and price on consumer responses", The Journal of Consumer Affairs, vol. 39, n.1, pp. 121-47.

MOHR L.A., WEBB D.J., HARRIS K.E. (2001), "Do consumers expect companies to be socially responsible? The impact of corporate social responsibility on buying behavior", The Journal of Consumer Affairs, vol. 35, n. 1, pp. 45-72.

NANDAM S. (2005), "An exploration of the brand identity-brand image linkage: a communication perspective", Journal of Brand Management, vol. 12, n. 4, pp. 264-78.

NEWHOLM T., SHAW D. (2007), "Studying the ethical consumer: a review of research", Journal of Consumer Behavior, vol. 6, n. 5, pp. 253-70.

OBERSEDER M., SCHLEGELMILCH B.B., GRUBER V. (2011), "Why don't consumers care about CSR? A qualitative study exploring the role of CSR in consumption decisions. Empirical paper", Journal of Business Ethics, vol. 104, n. 4, pp. 449-60.

OLSON J.C., REYNOLDS T.J.(1983), “Understanding consumers' cognitive structures: implications for advertising and strategy", in Percy L., Woodside A., Advertising and Consumer Psychology, Lexington Books, Lexington, Mass., pp. 77-90.

ORSINGHER C., MARZOCCHI G.L., VALENTINI S. (2011), "Consumer goal satisfaction: A means-end chain approach", Psychology and Marketing, vol. 28, n. 7, pp. 730-48.

PERRINI F., RUSSO A., TENCATI A., VURRO C. (2011), "Deconstructing the relationship between social and financial performance", Journal of Business Ethics, vol. 102, n. 1, pp. 59-76.

PETTY R.E., CACIOPPO J.T., STRATHMAN A.J., PRIESTER J.R. (2005) “To think or not to think? Exploring two routes to persuasion", in Brock T.C. and Green M.C., Persuasion: Psychological Insights and Perspectives, Sage Publications, Thousand Oaks, CA, pp. 81-116.

PLANK A., TEICHMANN K. (2018), "A facts panel on corporate social and environmental behavior: Decreasing information asymmetries between producers and consumers through product labeling", Journal of Cleaner Production, vol. 177, pp. 868-877.

PORTER M.E., KRAMER M.R. (2011), “Creating shared value”, Harvard Business Review, vol. 89, n. 1/2, pp. 62-77.

RASCHE A., MORSING M., MOON J. (eds.) (2017), Corporate Social Responsibility: strategy, communication, governance, Cambridge: Cambridge University Press.

ROBERTS J.A. (1996), "Will the real socially responsible consumer please step forward?”, Business Horizons, vol. 39, n. 1, pp. 79-83. 


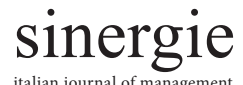
Vol. 37, Issue 3, 2019
ROKEACH M. (1973), The Nature of Human Values, Free Press, New York.

ROSEMBERG M. (1965), Society and the Adolescent Self-image, Princeton University Press, Princeton, NJ.

REYNOLDS T.J., GUTNAM J. (2001), "Laddering theory, method, analysis and interpretation”, in Reynolds T.J., Gutnam J. (eds.), Understanding Consumer Decision Making. The Means-End Approach to Marketing and Advertising Strategy, Lawrence Erlbaum Associates Publishers, Mahwah (N.J.).

RHODESN., WOOD W (1992), "Self-esteem and intelligence affect influenceability: the mediating role of message reception", Psychological Bulletin, vol. 111, $\mathrm{n}$. 1, pp. 156-71.

SEN S., BHATTACHARYA C.B. (2001), "Does doing good always lead to doing better? Consumer reactions to corporate social responsibility", Journal of Marketing Research, vol. 38, n. 2, pp. 225-43.

SIRGY J.M. (1982), "Self-concept in consumer behavior: a critical review”, Journal of Consumer Research, vol. 9, n. 3, pp. 287-300.

SIRGY J.M. (1985), "Using self-congruity and ideal congruity to predict purchase motivation", Journal of Business Research, vol. 13, n. 3, pp. 195-206.

SIRGY J.M., GREWAL D., MANGLEBURG T.F., PARK J., CHON K., CLAIBORNE C.B., JOHAR J.S., BERKMAN H. (1997), Assessing the predictive validity of two Methods of measuring self-image congruence", Academy of Marketing Science Journal, vol. 25, n. 3, p. 229.

TASSIELLO V., DE ANGELIS M., AMATULLI C. (2017), "Piattaforme digitali e rischio sociale nella condivisione di opinioni estreme", Micro\&Macro Marketing, n. 3, pp. 391-410.

TENCATI A., POGUTZ S. (2015), "Recognizing the limits: sustainable development, corporate sustainability and the need for innovative business paradigms", Sinergie, Italian Journal of Management, vol. 33, n. 96, pp. 37-55.

TEY Y.S., ARSIL P., BRINDAL M., LIEW S.Y., TEOCH C.T., TERANO R. (2018), "Personal values underlying ethnic food choice: Means-end evidence for Japanese food", Journal of Ethnic Foods, vol. 5, n. 1, pp. 151-69.

TRUMP R.K., NEWMAN K.P. (2017), "When do unethical brand perceptions spill over to competitors?", Marketing Letters, vol. 28, n. 2, pp. 219-230.

VICARI S. (1995), "Verso il Resource-Based Management", in Vicari S. (a cura di), Brand Equity. Il potenziale generativo della fiducia, Egea, Milano.

\section{Academic or professional position and contacts}

\section{Giuseppe Bertoli}

Full Professor of Management

University of Brescia - Italy
ISSN 0393-5108

pp. $149-170$

S I

MA

Italian Society of MANAGEMENT italian journal of management DOI $10.7433 / \mathrm{s} 110.2019 .07$
pp. $149-170$

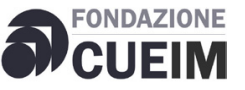

\section{Bruno Busacca}

Full Professor of Management

e-mail: bruno.busacca@unibocconi.it

\section{Alessandro Calzetti}

Sales Account - Gruppo Danone

e-mail. alessandro.calzetti93@gmail.com University Luigi Bocconi of Milano - Italy 